\title{
El mal a tolerar, el bien de tolerar, lo intolerable
}

\section{Introducción}

1.1.

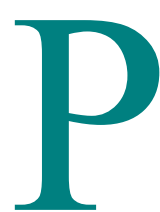

ara empezar esta pequeña contribución a un tema tan importante como el de la tolerancia, analizaré brevemente el verbo «tolerar» y los adjetivos «tolerante»e «intolerante», con el fin de llegar al significado, a una definición del término «tolerancia», o a la determinación del concepto de tolerancia. Antes, empero, es preciso ser consciente de la fuerza, tanto ilocutiva como perlocutiva, de algunos actos lingǘśsticos: actos mediante los cuales se reclama tolerancia y actos mediante los cuales se predica la tolerancia de un sujeto ${ }^{1}$.

Así, pues, en primer lugar tomaré en consideración un contexto reivindicativo, expresado de forma paradigmática en el enunciado

1. «S debe tolerar a $X »$,

$\mathrm{y}$, después, los contextos asertivos, expresados paradigmáticamente en los enunciados

2. «S es tolerante»

3. «S es intolerante»

4. «S tolera $a X »$

5. «S no tolera $a X »$

${ }^{1}$ Para la distinción entre significado y fuerza, así como entre fuerza locutiva, ilocutiva y perlocutiva de un acto lingüístico, ctr. J. L. Austin: How to Do Things with Words, Second Edition, J.O. Urmson and M Sbisa editors, Cambridge, Mass., Harvard University Press, 1962, Lecture VIII, pp. 94-108. Vease en p. 101-2 esta distinción: «Acto locutivo: me dijo «mátala»; acto ilocutivo: me ordenó matarla; acto perlocutivo: me persuadió para que la matase». 


\section{2.}

¿Qué informaciones mínimas transmite el enunciado 1.? ¿Qué contenidos comunica a quien no haya adquirido ningún conocimiento acerca de la situación particular, o contexto extralingüístico, en el cual, y con referencia al cual, tiene lugar el enunciado?

Como respuesta, pienso que hay que subrayar que, con el enunciado 1., X se coloca en la esfera de lo que es (o se piensa que es) distinto de S; entre tanto que el autor del propio enunciado se coloca más de la parte de $\mathrm{S}$ que de X: hasta llegar a identificarse con S (o, en el caso de que $\mathrm{S}$ sea un conjunto y no un individuo, colocándose en el ámbito de $\mathrm{S}$, pero presumiblemente en un sector particular de $\mathrm{S}$, el que mantiene las mejores relaciones con $\mathrm{X}$ ). De hecho, no se tiende a suponer que alguien pide ser tolerado; más bien se supone que alguien desea ser llamado tolerante; y, por tanto, o manifiesta su empeño en ser (o convertirse en) tolerante, o dirige la invitación al todo del que se considera parte para que sea tolerante.

De lo anterior se sigue que el receptor de la comunicación del enunciado L, se inclinará a pensar que, al menos en opinión del autor del enunciado, $\mathrm{S}$, al tolerar a $\mathrm{X}$, pasará a ser, en algún sentido, mejor.

Respondería, pues, a la primera pregunta sugiriendo que el enunciado 1., comunica, por presuposición, la alteridad [l'estraneità], que es una forma, aunque débil, de disvalor de X: alteridad desde el punto de vista de $\mathrm{S}$ y/o desde el punto de vista del autor del enunciado; al tiempo que comunica, por implicación, el valor para $\mathrm{S}$ de su tolerancia hacia $\mathrm{X}$.

\section{3.}

Paso, así, al examen de los contextos asertivos, tanto de contextos en los que la tolerancia se atribuye o se reconoce independientemente de circunstancias específicas y/o de objetos específicos, como de contextos en los cuales la tolerancia se atribuye o se reconoce en referencia a circunstancias específicas y/o a objetos específicos. Así, ¿qué se hace cuando se dice

2. "S es tolerante»

3. «S es intolerante»

4. «S tolera $a X »$

5. «S no tolera a $X »$ ?

A esta segunda pregunta debe responderse que los enunciados 2. y 3. seguramente se entienden como juicios de valor, pero no así los enunciados 4. y 5 . 
Con 2. se quiere expresar generalmente un juicio de valor positivo en relación a $\mathrm{S}$; con 3., un juicio de valor negativo en relación al mismo S. Debe subrayarse que no se trata de expresiones elípticas, ya que ambos juicios tienen sentido perfectamente aún sin la indicación del objeto o de los objetos tolerados ( $\mathrm{y}$, respectivamente, no tolerados) o considerados tolerables (y, respectivamente, intolerables).

Diciendo 4. y 5., en cambio, se puede, según el contexto, la intención y la situación, expresar un juicio de mero hecho, o un juicio de valor en relación a $S$; $y$, en la segunda hipótesis, cuál sea en cada uno de los dos casos el signo (positivo o negativo) del juicio de valor expresado, dependerá, además del valor que se atribuya o reconozca a $X$, también de factores diversos, que en cualquier caso enunciados como 4. o como 5. no especifican mínimamente. Esto significa que el juicio de valor, positivo en relación a la tolerancia y negativo en relación a la intolerancia, no se transfiere tal cual sobre tolerar y sobre no tolerar.

\section{4}

De este análisis de contextos, de este primer paso, obtengo el siguiente concepto de tolerancia, o definición del término «tolerancia»:

«Tolerancia es

(1) la actitud de individuos o grupos -sólo por metonimia puede predicarse de normas o de sistemas normativos-

(2) que consiste en relacionarse con otros (es decir, con individuos o conjuntos de individuos distintos o con expresiones y manifestaciones de individualidad percibidas como distintas -con sus comportamientos, actitudes, hábitos, creencias, opiniones, estilos de vida-).

(3) de un modo que se considera positivo genéricamente, pero no indiscriminadamente (o sea no cualquiera que sea el objeto sobre el que se declara),

(4) y que consiste en no asumir la diversidad (la alteridad [alteritá]) como un disvalor, en no negarla o rechazarla sólo porque sea tal;

(5) en cambio, no es elemento constitutivo del concepto, más allá de la caracterización negativa señalada en el punto (4), ninguna determinación positiva acerca del modo en que la actitud tolerante se desarrolle o manifieste».

Del concepto de tolerancia así establecido, destacaré -entre los múltiples que se podrían destacar- tres elementos constitutivos, que pienso pueden darse por adquiridos a estas alturas de la investigación, 
y a partir de los cuales intentaré construir la concepción de la tolerancia que es en mi opinión la mejor, sobre la base de los criterios que explicitaré2.

Antes, sin embargo, hay que delimitar el ámbito de la investigación: restringiré mi argumentación, y por tanto la concepción que voy a proponer, únicamente a la tolerancia llamada vertical. Es decir, tomaré en cuenta, de entre los individuos o conjunto de individuos de los que se puede predicar la tolerancia, exclusivamente aquellos dotados de alguna forma de poder visible y reconocido sobre los individuos respecto a los cuales (o respecto a cuyos comportamientos, actitudes, estilos de vida, opiniones, etc.) se declara la tolerancia o la intolerancia. La otra tolerancia, la tolerancia horizontal, no es evidentemente alternativa, sino complementaria a ésta, por lo que estaría fuera de lugar aquí hablar de concepciones; esta delimitación se aconseja simplemente por razones contingentes: por la imposibilidad de agotar, ni siquiera tocar en tan breve espacio, la variedad de problemas ligados al tema de la tolerancia ${ }^{3}$.

Por tanto -retomando el discurso- los tres elementos constitutivos del concepto de tolerancia sobre los que me basaré en lo que sigue son:

a) Que el objeto en relación al cual se reclama el ejercicio de tolerancia es siempre, aunque sea muy débilmente, cargado de disvalor.

${ }^{2}$ Para la distinción entre concepto y concepción es útil la distinción entre «noción»y «concepción» desarrollada por Ch. Perelman en «De la justice» (1945), in Ch. Perelman: Justice et raison, Bruxelles, Presses Universitaires de Bruxelles, 1963, pp. 9-80. En la p. 21: «Pour qu'une analyse logique de la notion de justice puisse constituer un progrès incontestable dans l'éclaircissement de cette idée confuse, il faut qu'elle parvienne à decrire d'une taçon précise ce qu'il y a de commun entre les conceptions opposées de la justice». Entre los autores que han utilizado esta distinción (prescindiendo de la influencia perelmaniana, que en algunos casos podría haberse dado a través de The Concept of Law de H.L.A. Hart-pero este no es el momento para tratar de documentarlo), cfr. W.B. Gallie: «Essentially Contested Concepts», Proceedings of Aristotelian Society, 1955-56, vol. 56, pp. 167-198; J. Rawls, A Theory of Justice, Cambridge, Mass., Harvard University Press, 1971, pp. 4-5 y p. 10; R. Dworking: Taking Rights Seriously, London, Duckworth, 1977, p. 103 y pp. 134-136; Ch. Swanton: «On the Essential Contestedness of Political Concepts», Ethics, 1985, vol. 95, n. 4, pp. 811-827; R. Dworkin: Law's Empire, London, Fontana Press, 1986, pp. 70-72 y pp. 90-101; P. Westen: Speaking of Equality. An Analysis of the Rhetorical Force of Equaluy in Moral and Legal Discourse, Princeton, Princeton University Press, 1990, pp.XVIII-XIX, pp. 48-49 y p. 124.

${ }^{3}$ Para la distinción entre tolerancia horizontal y vertical, ctr. E. Garzón Valdés: «No pongas tus sucias manos sobre Mozart. Algunas consideraciones sobre el concepto de tolerancia», Claves de razón práctica, 19, Enero-Febrero 1992, pp. 16-23. Garzón llama «vertical» a la tolerancia que presupone una relación de subordinación y «horizontal» a la que no la presupone. 
b) Que la tolerancia se valora siempre positivamente y la intolerancia negativamente.

c) Que no se atribuye la calificación de intolerante a todo aquel que no tolere a cualquiera; o sea, que se asume la legitimidad del juicio de intolerabilidad.

\section{Tres puntos de partida}

\section{1.}

El primer elemento, no particularmente original, puede considerarse trivial y por tanto legítimamente pertinente al concepto de tolerancia, y no a una peculiar concepción de la misma, sólo si la connotación de valor negativa de la que se habla, no se entiende como más fuerte de aquella intrínseca al concepto de «alteridad» [«estraneità»]. Se habrá notado que en el tratamiento precedente, con el fin de caracterizar el objeto de la tolerancia, el objeto a tolerar, he pasado del uso del término "otro», a través del uso del término «distinto», al del término "extraño» ${ }^{4}$; no lo he hecho de forma inadvertida o inocente: lo he hecho para que apareciera con la mayor fuerza posible lo que de negativo -que lo sea mucho o poco aquí no importa, ya que depende precisamente de las concepciones- contiene el propio concepto de otro. Pero en aquel momento de la investigación, no habría podido hacer más que caracterizar como extraño el objeto tolerado.

Hacer algo más, es decir, caracterizar resueltamente el objeto de la tolerancia como mal, o como amenaza (o como aquello, aquel, o aquellos que se perciben como mal o amenaza), constituye un paso, aunque frecuente, muy comprometido hacia una particular concepción de la tolerancia; paso que personalmente siento que debo dar, pero, al mismo tiempo, siento también que no debo esconder su importancia. Por eso hay que distinguir entre «tolerancia» e «indiferencia» ${ }^{5}$, así como

${ }^{4}$ Tanto «estraneità», como «alterità» se han traducido por «alteridad» porque el castellano carece de una sustantivación propia del adjetivo «extraño». No obstante, no parece haber mayores dificultades para comprender el texto [N. del T.].

${ }^{5}$ Se trata de una conocida distinción, pero en absoluto pacífica. Con la misma se abre, por ejemplo, la voz «Toleration» de la Encyclopedia of Philosophy. «Toleration is a policy of patient forberance in the presence of something which is disliked or disapproved of. Toleration must thus he distinguished from freedom or liberty, because it implies the existence of something believed to be disagreable or evil» (M. Cranston: «Toleration», in P. Edwards (ed.), Encyclopedia of Philosophy, vol. 8, London-New York, Macmillan, 1967, pp. 143-146, cita de p. 143). A título de ejemplo, además, puede mencionarse el siguiente pasaje: «To be tolerant involves the acceptance of 
entre «actitud tolerante» $\mathrm{y}$ «escepticismo» ${ }^{6}$; fijar las dos distinciones (que evidentemente no deben confundirse) $)^{7}$ comporta las opciones que se explicitarán.

Mi posición en esta sede es que distinguir entre tolerancia por un lado, e indiferencia y escepticismo por otro, impide ocultar que la tolerancia tiene costes que pueden ser muy elevados. En consecuencia, impide aceptar un planteamiento del tema susceptible de volver del todo inofensivo (para $\mathrm{S}$ ) y del todo inútil (para $\mathrm{X}$ ) el requerimiento de que $\mathrm{S}$ tolere a $\mathrm{X}$, de neutralizar sustancialmente la carga valorativa del juicio «S tolera a $\mathrm{X} », \mathrm{y}$, por consiguiente, la fuerza del acto lingüístico « $\mathrm{S}$ debe tolerar a $\mathrm{X} »^{8}$. Como ya se ha dicho, asumir una concepción de la tolerancia no indiferentista ni escéptica, no requiere pensar que el juicio de valor negativo sobre el objeto a tolerar sea realizado necesariamente por quien se pronuncia y/o se afana en favor de la tolerancia. De todos modos, ya hemos visto que está comprendido en el concepto de tolerancia el que aquél tenga relaciones privilegiadas con $\mathrm{S}$. Por lo que hace a las concepciones, podemos añadir que es necesario que

differences that really matter to you. If you do not care about people's conduct and preferences, then you are not tolerant in allowing them to do what they want, you are merely indifferent. Indifference and toleration should he clearly distinguished» (A. Weale: «Toleration, Individual Differences and Respect for Persons», in J. Horton and S Mendus (eds.), Aspects of Toleration, London-New York, Methuen, 1985 , p. 16, cursiva mía). Dicho esto, no cabe olvidar que la acusación de indiferencia dirigida al tolerante es la defensa típica del intolerante: Cfr. N. Bobbio: «Le ragioni della tolleranza», in N. Bobbio, L'età dei diritti, Torino, Einaudi, 1990, pp. 235-252, donde en la p. 237 se recuerda el aserto de B. Croce, según el cual los tolerantes «no siempre fueron los espíritus más nobles y heroicos. A menudo fueron los retóricos y los indiferentes. Los espíritus vigorosos mataban y se hacían matar» (B. Croce: Pagine sparse, Napoli, Ricciardi, 1943, vol. 11, p. 247).

${ }^{6}$ Esta distinción tiene un sentido diferente de la anterior, si bien ambas están conectadas. Tiene que ver con la problemática específica de la justificación de la tolerancia en el campo específico de la manifestación del pensamiento; quiere destacar -contra una extendida opinión- que el escepticismo no es condición necesaria ni suficiente de la tolerancia de las opiniones más dispares.

${ }^{7}$ No deben confundirse porque el escéptico puede ser totalmente indiferente respecto de la actitud, opinión, principio de acción, o teoría que no hace suya; mientras que el indiferente puede considerar que tiene los mejores argumentos de su parte, pero no ocuparse de ello.

8 Mi tesis según la cual la justificación escéptica de la tolerancia es susceptible de hacer inofensiva e inútil una reivindicación de tolerancia, expresa una posición próxima a la que considera contraproducente, es decir, un error estratégico, la adopción del escepticismo como fundamento último de la libertad de expresión: cfr. S.D. Smith: «Scepticism, Tolerance and Truth in the Theory of Free Expression», Southem California Law Review, 1987, vol. 60, pp. 649-731. 
asuma, al menos, el hecho de que en $\mathrm{S}$ se dan valoraciones expresadas en comportamientos $\mathrm{o}$ actitudes de censura o discriminación respecto a $\mathrm{X}$.

\section{2.}

El segundo elemento, siempre dentro de la construcción de una concepción de la tolerancia, pretendo asumirlo en un sentido muy fuerte. Implica que sea tenido de alguna manera como un bien para $\mathrm{S}$ la presencia en $\mathrm{S}$ de $\mathrm{X}$, aunque, se valore negativamente. Con tal interpretación de este componente del concepto de tolerancia, la reivindicación de la tolerancia se distingue de la simple exigencia de renuncia a (o abstención de) la represión'; por lo menos en cuanto la renuncia o la abstención (aunque se sustente en razones de principio más que de mera oportunidad ${ }^{10}$ ) comporte la decisión, o no excluya en línea de principio la legitimidad, del empleo de instrumentos de prevención alternativos a los represivos respecto a los comportamientos, o modos de pensar o de ser, desaprobados.

En virtud de esta misma interpretación, la concepción que propongo de la actitud tolerante se distingue también de la actitud de quien -sea individuo o grupo- busca el propio perfeccionamiento evitando eludir las pruebas, por duras que sean, que el entrar en contacto con el mal y/o el error le procuran. Según mi concepción, esta última es una actitud completamente diferente de la tolerancia; tan diferente como lo es una actitud religiosa de otra laica; o como lo es una actitud de superioridad de otra de paridad. El principio que lo inspira -al que llamaré «principio de sacrificio» ${ }^{11}$ «es totalmente distinto de mi «principio

${ }^{9}$ Así continua el párrafo de Weale citado en la nota 5: «The second condition to he satisfied is that those who are tolerant could get their way if they chose. If the state tolerates different ways of life, then this implies that it could restrict them if it chose. I shall mark this distinction in terms of difference between acquiescence and toleration. If the state cannot enforce legislation, as it was impossible to enforce prohibition in the United State, then I shall say that the state acquiesces in a course of action. If it could prevent it, but does not, then I shall say that it tolerates a course of action». Disiento de Weale tan sólo en que preferiría decir «Si el estado considera que puede, o que no puede, etc.» y no «Si el estado puede o no puede, etc.».

${ }^{10}$ Razones de principio serían, por ejemplo, las resumidas en el famoso «harm principle»; razones de mera oportunidad parecen ser, por ejemplo, las avanzadas por Locke en su justificación de la tolerancia: cir. J. Waldron: «Locke: Toleration and the Rationality of Persecution», in S. Mendus (ed.), Justifying Toleration. Conceptual and Historical Perspectives, Cambridge, Cambridge University Press, 1988, pp. 61-86.

${ }^{11}$ Además de «sacrificio», términos apropiados para indicar esta actitud, que considero muy importante distinguirla de la tolerancia, serían también «paciencia» y 
de tolerancia». En cambio, es parecido al «principio de indiferencia o de escepticismo»y al «principio de renuncia a la represión», pues -al igual que éstos- ni contiene ni expresa ningún interés en relación con aquello sobre lo que el principio mismo se declara, el cual permanece «otro», mero «objeto».

Interpretado de este modo, el segundo elemento no resulta en absoluto trivial. Proporciona una concepción que añade al concepto de tolerancia una especificación que excluía explícitamente el punto (5) de la definición mencionada, al introducir como elemento constitutivo esencial de la actitud tolerante el compromiso a una acción positiva tendente a superar la distancia, a colmar el hiato, que la diversidad comporta. Ello puede tener el efecto de hacer que el precio de la tolerancia sea aún más elevado, sobre todo si se mantiene la interpretación propuesta por el primer elemento, según el cual el objeto de la tolerancia es tal que suscita, en algún sentido, desaprobación.

\section{3.}

El tercer elemento, contrariamente a los dos primeros, es banal. Tiene que ver con la conocida problemática de los límites de la tolerancia, y aporta un dato que se da por descontado: la valoración positiva de la tolerancia no comporta en absoluto que todo deba ser tolerado ${ }^{12}$. Es obvio que hay que especificar los objetos en relación a los cuales ejercitar la tolerancia, de entre todos los posibles candidatos: todos los comportamientos, actitudes, inclinaciones, costumbres, características individuales, creencias, opiniones, que en algún sentido no gustan a alguien.

Pero si se da por descontado que la tolerancia tiene límites, los límites de lo tolerable, persiste, en cambio, la problemática acerca de cómo y dónde trazarlos; o sea, qué criterios usar si se quiere determinar

«aguante» [«sopportazione»]. Diría que es evidente que hay una diferencia entre la tolerancia, por un lado, y la paciencia o la capacidad (de temperamento o autoimpuesta) de aguante, por otro. En mi opinión, investigando el sentido de esta diferencia se llega a destacar el elemento positivo, construido a partir de una relación comunicativa con su objeto, que la actitud tolerante contiene.

${ }^{12}$ Le droit de la tolérance illimitée favorise les forts au détriment des taibles. La tolérance pour les violeurs signific l'intolérance pour les temmes. Si on tolere les tigres dans le meme enclos que les autres animaux, cela veut dire qu'on est pret a sacritier ceux-ci a ceux-la, ce qui est encore plus barbare et absurde. Les taibles, physiquement ou matériellement, sont les victimes de la tolérance illimitée; l'intolérance a l'égard de ceux qui les agressent est leur droit, non celui des torts» (T. Todorov: Les morales de l'histoire, Paris, Grasset, 1991, p. 209). 
la esfera de lo tolerable, y qué amplitud reconocer a esta esfera. En este tema, el interrogante más inquietante, en mi opinión, está en saber si el riesgo de la intolerancia se puede evitar más fácilmente fijando a la tolerancia limites de principio ${ }^{13}$ o límites de hecho ${ }^{14}$.

La concepción de la tolerancia que propongo admite que son necesarios límites de principio a la tolerancia, del mismo modo que es necesario que quien argumenta en favor de la tolerancia considere intolerable alguno de los objetos abstractamente concebibles de la tolerancia. Los conflictos con concepciones distintas de la tolerancia sobre este punto me parecen sólo aparentes, ya que opino que no debe sobrevalorarse la peculiaridad de esta caracterización de la tolerancia.

En cambio, considero digno de atención el punto relativo a la problemática de la legitimidad o ilegitimidad teórica de los límites de hecho a la tolerancia. Mi tesis es la de la ilegitimidad teórica de los límites de hecho a la tolerancia. En virtud de esta tesis, la concepción que propongo construye el principio de tolerancia como algo muy distinto de lo que llamaría el «principio de aquiescencia o de obediencia» ${ }^{15}$. «En base a este principio, el más peligroso de entre los hasta ahora

${ }^{13}$ La problemática de los límites de principio de la tolerancia es expresada, por ejemplo, en la ineludible y clásica pregunta: «¿Cómo combatir, y con qué armas, al representante de la intolerancia total? ¿Cómo pensar el problema de su libertad de expresión, y cómo definir la tolerancia hacia ésta, y qué hacer para que no se transforme en complicidad con el intolerante absoluto?» (J.-P. Faye: «Tolleranza/Intolleranza», in Enciclopedia, vol. XIV, Torino, Einaudi, 1981, pp. 292-317). El límite de principio más clásico a la tolerancia es justamente por definición la intolerancia. Lo que quiere decir que la intolerancia es intolerable. Por ejemplo, es un límite de principio el formulado por Locke contra los papistas: «Los papistas no deben disfrutar de los beneficios de la tolerancia, porque donde ellos tienen el poder, se consideran en la obligación de negarla a los demás. No es razonable que tenga plena libertad religiosa quien no reconoce como propio principio el que nadie debe perseguir o perjudicar a otro por el hecho de que éste disienta de él en un asunto religioso») (J. Locke,: Saggio sulla tolleranza, tr. it. in J. Locke: Scritti sulla tolleranza, vol. II, Torino, UTET, 1977, p. 111).

${ }^{14}$ El límite de hecho más clásico puede ser bien expresado con las palabras de Oliver W. Holmes, según el cual un principio guía para establecer si el hecho de proferir ciertas palabras ha de ser o no tolerado y protegido es «whether the words used are used in such circtunstances and are of such nature as to create a clear and present danger that they will bring about the substantive evils that Congress has a light to prevent. [...] When a nation is at war, many things that might be said in time of peace are such a hindrance to its effort that their utterance will not be endured so long as men fight and that no Court could regard them as protected by any constitutional right» (249 U.S., 1919, p. 52, cursiva mía).

${ }^{15}$ Sobre el peligro de que la tolerancia se transforme en obediencia y aquiescencia insiste L.C. Bollinger: The Tolerant Society, Oxford-New York, Clarendon Press, 1988,p.247:[...] the risk is that free speech will become a method of inculcating a kind of toleration that turns naturaly into passivity and uncritical obedience. While there is $[. .$.$] a definite and sometimes very great social need,$ in some 
diferenciados de la concepción ède tolerancia aquí propuesta (el principio de indiferencia, el principio de escepticismo, el principio de renuncia a -o de abstención de- la represión y el principio de sacrificio), pueden darse situaciones en los que, y para los que, la diversidad pierde sus eventuales connotaciones (o efectos) positivas/os para mantener únicamente los caracteres negativos, amenazadores. Entonces, puede suspenderse legítimamente la tolerancia, con la consecuencia de que la exigencia de asimilación o de consenso, incluso la limitación de derechos ${ }^{16}$, pueden pasar a ser legítimas.

Entre las que he mencionado, la concepción de la tolerancia como aquiescencia es, en mi opinión, la más peligrosa tanto objetivamente, como subjetivamente.

Es peligrosa objetivamente, en cuanto ella, más que neutralizar meramente la carga valorativa del juicio de tolerancia y la fuerza del acto lingüístico con el que se exige tolerancia (efecto producido, como he dicho antes, por la concepción de la tolerancia como indiferencia o escepticismo), las transforma respectivamente en una carga valorativa y en una fuerza de signo opuesto. Y a esta transformación se llega a través de una inversión, que no creo debida a una inadvertencia casual, de los papeles de los participantes en el «juego de la tolerancia»: el

context and on some occasions, for a noncritical, nonindependent mind, the aim of free speech is to work generally against that kind of intellectual character». Mi impresión, sin embargo, es que aquí Bollinger busca torpemente conciliar dos exigencias incompatibles: admitir la necesidad en ciertas situaciones, y por tanto la legitimidad, de la exigencia por parte de la sociedad de que los individuos asuman actitudes acríticas y heterodirigidas, se concilia mal con la defensa de una tolerancia que no sea ni obediente ni pasiva.

16 «Tal limitación ha asumido la forma extrema de la imposición de la segregación cuando, a partir de la primavera de 1942, alrededor de 112.000 personas de origen japonés, ciudadanos americanos o no, residentes en la costa occidental de los Estados Unidos, se les impuso el toque de queda y se ordenó su evacuación. El episodio fue definido inmediatamente como «the most dramatic invasion of the rights of citizens of the United States by their own government that has thus far occurred in the [nation's] history» (E. S. Corwin: Total War and Constitution, 1947, p. 91, cit. in S. L. Paulson: «Material and Formal Authorisation in Kelsen's Pure Theory», The Cambridge Law Journal, vol. 39, 1980, pp. 172-193, cita en p. 180); actualmente siguen las referencias a este acontecimiento calificado «as the most massive violations of civil liberties in U.S. history» (M. Linfield: Freedom Under Fire. US. Civil Liberties in Times of War, Boston, Mass., South End Press, 1990, p. 95. En el fallo Korematsu v. United States, 323 U.S.S. 214 (1944), el Tribunal Supremo de los Estados Unidos afirmó: «Korematsu was not excluded from the Military Arca hecause of hostility to him or his race. He was excluded because we are at war with the Japanise Empire, because the properly constituted military authorities feared an invasion of our West Coast and felt constrained to take proper security measures» (p. 223). 
poder deviene objeto y el individuo (ahora tal vez convertido en súbdito), sujeto. Como ha sido ejemplificado agudamente, dentro de esta lógica ¿quién es ya tolerante del católico que acepta el dogma de la infalibilidad del Papa? ${ }^{17}$

$\mathrm{Y}$ es peligrosa contingentemente, porque la concepción de la tolerancia como obediencia o aquiescencia es de la que puede ser presa más fácil nuestra sociedad, caracterizada al mismo tiempo por un escepticismo difuso y por una extraordinaria capacidad de control de la opinión pública por parte de los medios. Los medios de comunicación están en condiciones de manipular el flujo de informaciones, así como -mediante un uso del lenguaje (de los varios lenguajes) no comunicativo, sino estratégico- guiar intereses, suscitar actitudes, opiniones, entusiasmos, indignaciones, en definitiva producir consenso ${ }^{18}$.

En sociedades de este tipo es posible y fácil, y desgraciadamente es trágica experiencia cotidiana, que, invirtiendo la inspiración fundamental ético-política del concepto, la tolerancia se invoque contra la crítica, de tal modo que la admisión de la legitimidad de los límites factuales, se transforma fácilmente en la admisión de la ilegitimidad de límites de principio $^{19}$.

Siguiendo el hilo de la argumentación determinada por los tres elementos citados, dividiré lo que resta en tres momentos que llamaré: el mal a tolerar, el bien de tolerar, lo intolerable.

\section{EI mal a tolerar}

Quedando claro, por la concepción aquí propuesta, que el objeto para el que se reivindica tolerancia es siempre -de alguna manera- cargado de disvalor (por el autor de la reivindicación y/o por el sujeto que se pretende tolerante, o por un componente de éste), quedan por explicitar algunas consecuencias de tal posición.

La primera es que, si se quiere mantener hoy, en nuestras sociedades, la fuerza de la reivindicación de tolerancia, no puede ligarse

${ }^{17}$ Cfr. L.C. Bollinger, op. cit, p. 246.

${ }^{18}$ Cfr. H. Marcuse: «La tolerancia represiva», in R.P. Wolff, B. Moore jr., H. Marcuse: Critica della tolleranza. La forma attuale della tolleranza: un mascheramento della repressione, tr. it. de D. Codelli, Torino, Einaudi, 1968, pp. 77- 105.

${ }^{19}$ Una de las tareas fundamentales de la crítica es la de recordar a quienes detentan el poder el respeto a los principios, y, por tanto, si es el caso, también el respeto a los límites de principio a la tolerancia. Pero al rebautizar a la critica como «moralismo», «dogmatismo», «intolerancia» se consigue transferir sobre la crítica, mediante una definición persuasiva implícita, la connotación de valor negativa generalmente atribuida al concepto de intolerancia. 
la problemática de ésta con la de la verdad, pidiendo tolerancia para la no verdad -es decir, según los casos, para el error ${ }^{20}$, o para la opinión ${ }^{21}$ - o también para las verdades no demostrables, como las de los postulados ${ }^{22}$.

En efecto, creo que hay que reconocer que, en relación con la no verdad (ya se presente en la forma del error o de la opinión), nuestra

${ }^{20}$ La tolerancia para el error es la que se reclama desarrollando la argumentación, típica de los siglos XVII y XVIII, de apoyo a la libertad de discusión y de investigación; argumentación según la cual sólo la comparación con el error puede hacer resaltar la fuerza de la verdad. Cfr., por ejemplo, C.A. Helvétius: De l'Homme, Secc. IV, cap. XV: «Du moment oú le citoyen pourra tout penser, tout écrire, que de livres faits sur des matieries qu'il n'entendra pas! Que de sottises les écrivains ne diront-ils pas! Tant mieux: ils en laisseront moins a faire aux Visirs. La critique relevera les erreurs de Pauteur: le public s'en moquera; c'est toute la punition qu'il mérite. [...] En quelque gente que ce soit, un excellent livre en suposse une infinité de mauvais». El de la fuerza de la verdad que únicamente puede resplandecer comparándola con el error es, como es sabido, un argumento que sobrevive en pleno siglo XIX, en las páginas del segundo capítulo de On Liberty de J.S. Mill, aunque sea originando, entre ésas y otras páginas fundamentales del texto, una incongruencia difícil de resolver: cfr. nota siguiente.

${ }^{21}$ La justificación no cognitivista, o incluso relativista, de la tolerancia es la más común en el siglo XIX, y consiste en unir estrechamente el valor de la tolerancia liberal a la tesis epistemológica de la distinción entre juicios de hecho (y juicios analíticos) y juicios de valor. Cfr., por ejemplo, Ch. Perelman: «De la méthode analytique en philosophie», in Ch. Perelman, Justice et Raison, cit, pp. 81-94; en la p. 89: «[...] ma conception philosophique considre les jugements de vérité comme n'admettant pas de contradiction et [... ] leur oppose des jugements de valeur qui non seulement tolrent, mais même supposent l'existence de jugements de valeur opposés. A l'unité de la vérité, j'oppose le pluralisme des valeurs se trouvant a la base de la tolérance que l'on devrait témoigner à l'égard des jugements de valeur». Como es sabido, esta misma línea argumentativa, aunque no formulada exactamente así, se encuentra ya en el siglo XIX en el tercer capítulo de On Liberty de J.S. Mill, donde se produce la incongruencia (de la que se habla en la nota anterior) con el segundo capítulo de la misma obra; sobre esto, cfr. D. Edwards: «Toleration and Mill's Liberty of Thought and Discussiorn», in S. Mendus (ed.): Justiffing Toleration, cit., pp. 87-113. En ocasiones, esta línea argumentativa se transforma en una justificación pragmática del no cognitivismo o del relativismo a partir del valor de la tolerancia; cfr., por ejemplo, lo que escribe U. Scarpelli a propósito de la Gran División entre is y ought, también llamada «ley de Hume» En mis reflexiones hoy ya lejanas [...] fui llevado [...] a buscar por la propia ley de Hume un fundamento ontológico [...] Hoy, en cambio, tiendo a invertir los términos, concluyendo que debemos aceptar la ley de Hume no ya debido a que el hombre es libre (y la ley de Hume respeta su libertad), sino porque queremos hacerlo libre, porque tenemos en nuestra ética el valor de la libertad». (U. Scarpelli: «La meta-etica analitica e la su rilevanza etica», in U. Scarpelli, L'etica senza verità, Bologna, II Mulino, 1982, p. 110).

${ }^{22} \mathrm{El}$ «principio de tolerancia» es, como se sabe, la importante tesis de R. Camap, según el cual la ciencia -en oposición a toda metafísica- se explica como un continuo desarrollo de postulados convencionales y de hipótesis, utilizables de distintas formas, y se configura como un proceso autocorrectivo (cfr. R. Camap: Foundations of Logic and Mathematics, Chicago, 1939). 
cultura en general manifiesta más indiferencia que rechazo, o simplemente molestia.

La pluralidad de creencias, opiniones e incluso explicaciones científicas de un mismo fenómeno, son datos hoy ya consolidados, que no tienen necesidad alguna de ser tolerados. De este modo, insistir particularmente, o, sin más, limitarse a ellos, en un tratamiento general sobre la tolerancia que no se plantee prevalentemente como investigación histórica, puede tener el efecto ya dicho de neutralizar la función de reivindicación de la tolerancia. Además, puede conducir a la idea injustificada de que la pérdida de centralidad del valor de la verdad en una cultura ha de ir necesariamente de la mano, o ser lo mismo, que la conquista de la tolerancia. Y pensar por ello que actualmente ya se ha alcanzado la victoria en la batalla por la tolerancia ${ }^{23}$.

En mi opinión, esto es falso. Es cierto que hoy, por ejemplo, en los países occidentales la tolerancia expresada en el reconocimiento de la libertad de pensamiento, de investigación, de consciencia y de culto no es, en línea de principio, ${ }^{24}$ puesta en duda. Pero la intolerancia no se expresa sólo en la censura, sino también en el prejuicio, y, por tanto, en la discriminación ${ }^{25}$.

${ }^{23}$ «This failure to distinguish tolerance from manifestation of uncertainty is a prevalent confusion. It is often remarqued that ours is a tolerant age [...] In fact, this openness to divergent views stems less from a mien of tolerance than from the endemic lack of conviction; having trascended the 'age of belief' we are left without heresies as well» (J. Halberstam: «The Paradox of Tolerance», The Philosophical Forum, vol. 14, 1982-83, p. 195, cit., in S.D. Smith: «Skepticism, Tolerance, and Truth in the Theory of Free Expression», cit., p. 653).

${ }^{24}$ Digo en línea de principio porque en los hechos, como se sabe, las cosas pueden funcionar, y a veces funcionan, de un modo bastante distinto. Pero lo que aquí hay que notar es que, donde eso suceda, el paso que hay que dar consiste en una apelación al derecho positivo, en la denuncia de una violación puntual de normas válidas, normas que en la mayor parte de los casos, hasta forman parte de los principios fundamentales contenidos en las Constituciones jurídicas de las sociedades en cuestión. No es, pues, necesario, en estos casos, una genérica apelación a la tolerancia política, siendo suficiente (al menos para la legitimidad, si no también necesariamente para la eficacia del paso) una llamada específica a normas jurídicas. Cfr. E. Garzón Valdés: «No pongas tus sucias manos sobre Mozart», cit., p. 21: «... en los países occidentales no es que se «tolere» la libertad de cultos sino que ella es un derecho de todo ciudadano. La tolerancia tiene una cierta connotación de «condescendencia» o «disimulo», como decía Francisco Suárez; ello no vale en modo alguno para las permisiones o autorizaciones, por más que en su genealogía figuren actos de tolerancia».

${ }^{25}$ Cfr. L-C. Bollinger, op. cit., pp. 111-112. En sentido contrario parece haberse expresado recientemente N. Bobbio: «Le ragioni della toleranza», cit., pp. 235-236. Digo «parece» porque, a pesar de la interpretación dada por A. E. Galeotti: «La questione della tolleranza», prepint de Politeia. Contributi per la discussione, 1990, n. 36, no estoy segura que las razones ofrecidas por Bobbio en aquellas páginas para 
Por otra parte, aún permaneciendo dentro del campo, limitado pero de gran relevancia, de la libertad de expresión, es preciso subrayar que puede considerarse expresión de intolerancia no sólo impedir la transmisión de ciertos mensajes. Puede ser expresión de intolerancia también la transmisión de ciertos mensajes (además de eventualmente su contenido) procedentes de grandes órganos de información sin competencia. Y ello a pesar de que la crítica no sea declarada inadmisible, si la oposición es técnicamente imposible, o, siendo posible, se realiza siempre demasiado tarde, cuando el efecto del mensaje, su impacto sobre la opinión pública ya ha tenido lugar ${ }^{26}$.

Al final del camino que empieza con la asunción de una actitud tolerante está efectivamente la libertad, pero también el reconocimiento de la igualdad ${ }^{27}$. De ello resulta que, hoy, la reivindicación de tolerancia

distinguir el problema de la convivencia de creencias diversas del de la convivencia con los distintos, se entiendan o deban entenderse también como razones para negar legitimidad a la que el propio autor reconoce que es una extensión actual del concepto que atañe también al segundo tipo de situaciones y problemas. En efecto, escribe Bobbio: «Cuando se habla de tolerancia en su significado históricamente prevalente, nos referimos al problema de la convivencia de creencias [...] Hoy el concepto de tolerancia se extiende al problema de la convivencia de las minorías...» (op. cit., p. 235). Entre las dos situaciones, continúa, hay una diferencia: «La mejor prueba de esta diferencia está en el hecho de que en el segundo caso la expresión habitual con la que se designa lo que se debe combatir, incluso en los documentos oficiales internacionales, no es la intolerancia, sino la discriminación, sea racial, sexual, étnica, etc. (op. cit., 236). En relación a las dos citas quisiera hacer dos precisiones, con el fin de mostrar cómo en ninguna de ellas descubro argumentos contra mi propuesta teórica. La primera es que el hecho de que el significado histórico prevalente del término sea de un cierto tipo es una premisa de mi argumentación, según la cual precisamente sobre este significado hoy se debe trabajar críticamente para que la reivindicación actual de tolerancia no pierda la fuerza que históricamente siempre ha tenido. La segunda es que «intolerancia» y «discriminación» me parecen términos que pertenecen a contextos y a universos de discurso distintos: el primero al universo de la teoría política, el segundo al de la teoría del derecho; por ello, por ejemplo, me parecería razonable decir que la intolerancia es la razón y/o la causa de la discriminación.

${ }^{26} \ll \mathrm{Si}$ je lis aujourd'hui un article dans le journal, insinuant que les femmes n'aiment rien autant que la souffrance physique et moral dont elles pourraient devenir l'objet, dois-je le considérer, avant tout, comme une recherche de vérité (celle de l'essence léminine) ou comme une incitation à l'action (il est normal que les hommes battent leur femmes)?» (T. Todorov, op. cit., p. 201). Acerca de la problemática muy importante de la fuerza de los actos lingüisticos en relación a la garantía de la libertad de expresión dada por la Primera Enmienda de la Constitución de los Estados Unidos, cfr. K. Greenawalt: Crime, Speech and the Uses of Language, New York, Oxford University Press, 1989.

${ }^{27}$ Cfr. A. Weale: «Toleration, Individual Differences and Respect for Persons», cit., p. 29: «To provide a general argument for toleration we need a concept of equal respect. That is, persons have to respect one another as equals, this respect implying 
se entiende como exigencia no sólo de no impedimento, sino también de igual oportunidad y de igual tutela ${ }^{28}$.

Este planteamiento comporta sin duda la puesta entre paréntesis de una característica de la tolerancia que alguno podría considerar esencial, incluso un elemento de la propia definición del término «tolerancia». Sería la característica de la pasividad, según la cual la actitud de tolerar excluye (y no sólo no comporta necesariamente) un actuar, y comporta únicamente (según la etimología latina del término) un soportar. Pienso que aquí las razones filológicas deben ceder frente a las históricas y las de filosofía política. Si el término «tolerancia», con sus precedentes, nos remite sin duda también a aquel significado, no todos los usos del mismo lo mantienen como elemento relevante. Y este es el caso de las teorías políticas modernas a las que me refiero.

Es cierto que históricamente el discurso sobre la tolerancia se ha insertado más en el de la libertad negativa que en el de la libertad positiva ${ }^{29}$. Pero no lo es menos, por ejemplo, que la clásica manifestación de tolerancia política que consiste en el reconocimiento de la libertad de expresión, requiere un actuar además de un soportar, requiere el establecimiento de las condiciones adecuadas para permitir su ejercicio, así como las condiciones necesarias para que la palabra hablada pueda ser también oída; sin contar con que las justificaciones tenidas en cuenta habitualmente para esta forma de tolerancia se fundan en el concepto de democracia entendida como participación, y, por tanto, se refieren más a la libertad de los antiguos que a la de los modernos ${ }^{30}$.

that each person recognizes others as capable and competent to form their own projects and plans of life». Cfr. también M.Minow: «Putting Down: Tolerance Reconsidered», Osgoode Hall Law Journal, 1990, vol. 28, pp. 409-448.

${ }_{28}$ Cfr. A. Weale: «Toleration, Individual Differences and Respect for Persons», cit., pp. 31-32, donde se cita el caso de un individuo que viva en una sociedad secularizada y que, sin embargo, desee abstenerse de trabajar un día que considera santo. Está claro que no podrá pretender, amparándose en el principio de igual respeto para las personas asumido como justificación de la tolerancia, que el Estado reconozca aquel día como una jornada de descanso para todos. No obstante, el Estado podrá, por respeto al mismo principio, tomar las medidas positivas de tutela de las minorías; por ejemplo, solicitar a los empresarios que adapten, en la medida de lo posible, los horarios y los hábitos laborales a las peticiones de los individuos y de los grupos.

${ }^{29}$ Es más, la teoría liberal de la tolerancia se ha identificado a veces con la concepción del Estado-guardián nocturno, y ambas, con las teorías de la neutralidad del Estado en relación con cualquier concepción del bien.

${ }^{30}$ Cfr., por ejemplo, A. Meiklejohn: «Free Speech and Its Relations to Self-Government», in Political Freedom: The Constitutional Powers of the People, $2^{\mathrm{a}}$ ed., New York, Oxford University Press, 1965, p. 24: ... Now in that method of political self-government [i.e. that of the traditional New England town meeting] the point of 
La tolerancia, pues, no es mera indulgencia, ni tampoco, como ya he dicho, mera renuncia a la represión. La tolerancia comporta efectivamente abstenerse de reprimir, pero consiste en un abstenerse de reprimir más una actividad promocional, al contrario que un abstenerse de reprimir más una actividad de prevención.

Indudablemente se da una paradoja en la tolerancia: ¿no es esquizofrénico un planteamiento que se ejerce sobre un objeto juzgado de alguna forma negativamente, y que, al mismo tiempo, comporta que en relación a ese objeto se ejerza una actividad, en algún sentido, promocional? ¿Cómo es posible establecer que lo que es un mal se incentive?

No se trata de esquizofrenia, sino de pluralismo ${ }^{31}$. La sociedad tolerante es una sociedad pluralista, crítica en relación a los propios valores y sobre todo en relación a la propia idiosincrasia; una sociedad que se pone en discusión y desea su propia transformación, interpretada como maduración. Por eso la tolerancia reclamada se considera productiva para la sociedad, de consecuencias benéficas, ya que se entiende que acelera -considerándolo como madurez- el mismo proceso de transformación que expresa.

\section{El bien de tolerar}

Así, pasamos al segundo elemento, que consiste en el reconocimiento del hecho de que la tolerancia se valora siempre positivamente y la intolerancia negativamente. Tolerancia quiere decir disponibilidad a la comparación; quiere decir reconocimiento y aceptación de la diferencia advertida como desafío y, como tal, considerada estimulante más que amenazadora.

Propongo, pues, considerar fundamental en la actitud tolerante el reconocimiento de una misma naturaleza en el sujeto y en el objeto de la tolerancia: el sujeto que tolera se define, implícitamente, como parte del mismo todo que comprende el objeto a tolerar ${ }^{32}$. Por tanto, al definir

ultimate interest is not the words of the speakers, but the minds of the hearers. The final aim of the meeting is the voting of wisw decisions. The welfare of the community requires that those who decide issues shall understand them. They must know what they are voting about. And this, in turn, requires that so far as time allows, all facts and interests relevant to the problem shall be fully and fairly presented to the meeting».

${ }^{31}$ Cfr. J. Raz: «Autonomy, Toleration, and the Harm Principle», in S. Mendus (ed.), Justifying Toleration, cit., pp. 155-175.

32, Cfr. T. Todorov, op. cit., p. 191-192: «A l'opposé d'une vision traditionaliste de l'humanité, qui veut que nous soyons les seuls etres humains ou en tout cas la meilleure incamation de l'humanitá, et que les autres soient d'autant moins humains qu'ils habitent plus loin de nos frontires, ceux qui croient à l'universalité du genre 
la relación de tolerancia se toman en consideración tres pertenencias distintas pero conectadas: la pertenencia del sujeto tolerante a un conjunto, la pertenencia del destinatario de la tolerancia a un conjunto distinto y la común pertenencia de ambos a un conjunto más amplio, es decir, la colocación entre ambos de una estructura de referencia superior capaz de contenerlos, en relación a la cual también los elementos de los dos conjuntos parciales pueden (y, a ciertos fines, deben) ser definidos.

Desde este punto de vista, tiene sentido considerar siempre la asunción del hábito de la tolerancia como la asunción de un riesgo; como la decisión de tomar un camino de trayecto y meta, al menos en parte, imprevisibles; como cumplimiento de un primer paso, bien sea por la vía del reconocimiento positivo de la diferencia, bien sea por la de la integración de un sistema en el otro, o de una fusión entre los dos sistemas. Al término del trayecto (que de todos modos puede que nunca se siga hasta el final) puede acontecer perfectamente, debido a los elementos constitutivos de los dos conjuntos parciales, la pérdida de las connotaciones de las recíprocas pertenencias primarias ${ }^{33}$.

Evidentemente, que la tolerancia pueda considerarse un primer paso, no significa que no sea una meta muy importante a la que se llega reconociendo al destinatario de la tolerancia la misma naturaleza del sujeto.

Del hecho de conectar de este modo la problemática de la tolerancia a la del pluralismo se deduce que, permaneciendo inmutable la tolerancia en cuanto a actitud, los límites, en cambio, son tendencialmente móviles. La idea de tolerancia se vincula a la de transformación, en el

humain sont préts à tolérer les diffiérences qu'i1s observent chez les ressortissants d'autres pays. [...] L'un des ingrédients de cette impulsion est la découverte de nouveaux mondes, et donc la prise en considération d'une diversité humaine beaucoup plus grande que celle qui était connue jusqu'alors». Sobre esta problemática, cfr. S. Landucci: I filosofi e i selvaggi 1580-1780, Bari, Laterza, 1972.

${ }^{33}$ Según A.E. Galeotti: «La questione della tolleranza», cit., pp. 33-34, dado el problema de la «existencia de grupos que se distinguen entre sí por una diferencia desagradable para la mayoría (y desagradable en cuanto se halla conectada al poder de amenaza del propio grupo), la solución propuesta por la tolerancia es la coexistencia pacífica de grupos distintos reconocidos dignos de igual respeto y coparticipantes en la arena política con igual dignidad. El igual respeto al que tienen derecho las minorías se presta, sin embargo, a dos interpretaciones: la primera, y más fuerte, mantiene que la base del igual respeto está en un reconocimiento positivo de la diferencia, factor de la preexistente intolerancia. [...] La segunda interpretación, más débil, sostiene que el igual respeto atribuido a la minoría depende, en cambio, del hecho de decolorar la diferencia, hasta que el grupo aparezca igual a la mayoría. La diferencia, por tanto, se legitima públicamente, pero en cuanto no impide la dignidad y el respeto al que todo ser humano tiene derecho. El acceso a una igual ciudadanía se pagaría así en términos de tendencial asimilación y, en última instancia, homologación». 
sentido de progresiva superación de los prejuicios, ampliación de horizontes, apertura de nuevas perspectivas: en el sendero que, idealmente, lleva a mirar «a ninguna parte» ${ }^{34}$

Sobre la base de esta concepción de la tolerancia no siempre es necesario distinguir dos sistemas normativos o valorativos de referencia, uno de los cuales valore negativamente y el otro positivamente (o provea razones para no reprimir) el objeto a tolerar ${ }^{35}$. En mi opinión, la paradoja de la tolerancia -que he intentado expresar mediante las dos locuciones «el mal a tolerar» $\mathrm{y}$ 《el bien de tolerar»- puede resolverse, de manera quizás menos artificial que cuando se divide en dos el sistema axiológico de referencia, introduciendo en la argumentación la perspectiva diacrónica y el concepto de «transformación».

El expediente de la duplicación del sistema axiológico de referencia me parece necesario únicamente a partir de la asunción de una perspectiva según la cual los sistemas éticos serían susceptibles de ser bien representados según el modelo kelseniano de «sistema normativo estático». Serían estructuras deductivas, y por tanto, por definición, coherentes, procedentes de un único principio supremo, de una noma fundamental injustificada e injustificable (arbitraria) ${ }^{36}$. Si los sistemas éticos fuesen de este tipo, sería evidentemente inconcebible que un único sistema contuviese la norma en base a la cual, por ejemplo, no debe realizarse cierto comportamiento, y, junto a ella, la norma en base a la cual debe realizarse el mismo comportamiento.

Pero, en mi opinión, los sistemas éticos pueden representarse mejor como estructuras normativas complejas que se forman durante los procedimientos de justificación de juicios morales. Esos procedimientos van desde los juicios a los principios más que a la inversa y no necesariamente conducen todos a un único principio, sino a diversos,

${ }^{34}$ Cfr. Th. Nagel: The View from Nowhere, New York, Oxford University Press, 1986.

${ }^{35}$ En relación a la tolerancia, E. Garzón Valdés considera siempre necesario establecer esta distinción: «No pongas tus sucias manos sobre Mozart», cit., pp. 18-19, donde se distingue entre sistema normativo básico y sistema normativo justificante: «La tolerancia está doblemente referida a sistemas normativos. Por una parte, la tendencia a no permitir el acto finalmente tolerado proviene del sistema a cuya regulación está sujeto. Llamaré a este sistema «sistema normativo básico». Por otra, la superación de esta tendencia resulta de la consideración de principios y/o reglas de un sistema normativo superior. Llamaré a este sistema "sistema normativo justificante». Para Garzón, sin embargo, no se trata de dos sistemas éticos, sino de un sistema moral (justificante) y de un sistema de normas de otra especie -de etiqueta, religiosas, etc.- (básico).

${ }^{36}$ Cfr. H. KELSEN: Reine Rechtslehre. Mit einem Anhang: Das Problem der Gerechtigkeit, Wien, Deuticke, 1960, cap. V; L. Gianformaggio (ed.): Sistemi normativi staticie dinamici. Analisi di una dicotomia kelseniana, Torino, Giappichelli, 1991. 
no necesariamente jerarquizables en un conjunto formalmente coherente, sino sólo, y aún entonces con alguna dificultad, armonizables en un conjunto sustancialmente congruente.

Que tales principios sean no justificados, es, desde el punto de vista de los procedimientos individuales de justificación de los que aquéllos constituyen el punto de llegada, una necesidad lógica; desde el punto de vista del sistema ético considerado en perspectiva sincrónica, es verdad por definición; desde el punto de vista del sistema ético considerado en perspectiva diacrónica y contingente, es probablemente falso.

Que se trate de principios no justificables no tiene sentido. La evolución o transformación de una moral (que no quiere decir sustitución de un sistema moral por otro) toma cuerpo a menudo, o tal vez en la mayoría de los casos, precisamente mediante la exigencia de justificación de principios considerados hasta entonces no necesitados de justificación alguna. Y la exigencia surge a partir de reflexiones originadas por experiencias nuevas. Procede de quien empieza a notar demasiado limitada la protección ofrecida por ciertos principios (por ejemplo, por el principio según el cual no deben matarse seres humanos pertenecientes a la propia raza, o, más tarde, por aquel según el cual no deben matarse individuos de la especie humana). Pero también procede de quien empieza a ver la protección ofrecida por ciertos principios como demasiado amplia (por ejemplo, por el principio según el cual, en cualquier situación y de cualquier modo, se debe perseguir el objetivo de la prolongación de la vida de un ser humano).

En mi opinión, la cuestión de la tolerancia aparece típicamente en estas fases de transformación, en las que en la sociedad se considera legítimo interrogarse respecto a la justificación de principios con anterioridad considerados supremos; y antes aún de que se hable de reconocimiento de derechos ${ }^{37}$, se reclama, a quien detenta el poder, el ejercicio de tolerancia (violando a veces también normas positivas) ${ }^{38}$.

${ }^{37}$ Aquí es importante recordar que el objeto de estas páginas es la tolerancia que, adoptando la terminologia de Garzón Valdés, he llamado «vertical», y no la «horizontal». En efecto, en este segundo sentido de tolerancia es muy posible, y en Europa es hoy trágica experiencia cotidiana, que el Estado y algunos sectores de la sociedad consideren necesario reclamar a otros sectores el ejercicio de tolerancia, en relación a objetos acerca de los que ya se ha reconocido la plena y formal tutela jurídica: casi cada día, como es sabido, nos informan sobre «episodios de intolerancia racial» y sobre «manifestaciones en favor de la tolerancia racial».

${ }^{38} \mathrm{Si}$ bien seguramente está deformado por los usos lingüísticos el que se califique como acto de tolerancia (obviamente, como acabamos de ver, en el sentido de la tolerancia llamada «vertical») la verificación del hecho de que ha sido ejercido un derecho previamente atribuido por una norma, no me parece que suceda lo mismo con la calificación como acto de tolerancia de la eventual inaplicación de una norma competente para aplicar. Expresa una opinión distinta A. Schmitt: «Las circunstancias de la tolerancia», en este mismo número de DOXA. 
Como conclusión de este apartado se puede concordar con Peter Nicholson cuando dice que la tolerancia es un valor moral: «By being required to take heed of the ideas of others, whether simply as opinions or as embodied in their lifestyles, and above all when one forces oneself to do so, one is schooled and educated. One is shown that part of being moral, and of treating other moral agents morally, is to give serious consideration to their ideas ${ }^{39}$. La tolerancia es un valor moral porque deriva, y es exigencia, del valor moral de la persona: «The moral ideal of toleration does not require the tolerator to acknowledge any merit in the opinions of which he disapproves; but he must respect the personality of the holders of those opinions, and treat them as moral agents whose views can be discussed and disputed, and who, are capable of changing their minds on rational grounds ${ }^{40}$.

La atribución de igual valor moral a toda persona -sobre la que se fundamenta la tolerancia- no comporta, pues, la aceptación de cualquier valor o actitud asumidos por cualquier persona. Hay evidentemente límites. Pero, ¿cuáles? ¿Hasta qué punto la tolerancia requiere que se respeten las opiniones y se protejan los estilos de vida de las personas?

Piénsese, por ejemplo, en el racismo. El fenómeno «racismo», que sobre la base del concepto de tolerancia aquí propuesto puede calificarse como manifestación paradigmática de intolerancia ${ }^{41}$, determina también una constelación de posibles objetos de tolerancia o intolerancia ${ }^{42}$. La eventual admisión de que, en ciertos casos, en nombre de la «tolerancia»

${ }^{39}$ P.P. Nicholson: «Toleration as Moral Ideal», in J. Horton and S. Mendus (eds.), Aspects of Toleration, cit., pp. 158-173, cita en la p. 165.

${ }^{40}$ P.P. Nicholson: «Toleration as Moral Ideal», cit., pp. 165- 166.

${ }^{41}$ «El racismo es la doctrina que ve en las diferencias raciales la causa fundamental del devenir histórico, y que, ordenando las diversas razas en una jerarquía de valores, afirma para aquellas que se pretenden superiores, el derecho a gobernar el mundo y de tener a las inferiores en una posición subordinada, sin descartar, llegado el caso, la hipótesis de su eliminación» (B. Salza: «Razzismo», in N. Bobbio e N. Matteucci: Dizionario di politica, Torino, UTET, 1976, pp. 845-849, cita en las pp. 845-846).

${ }^{42}$ Sobre el problema de la difícil conciliación entre la tutela de la libertad de expresión y la tutela de la igual dignidad de las personas mediante la sanción del uso de locuciones verbales que expresen, o inciten al, odio racial, cfr. A. Callinicos: «Repressive Toleration Revisited: MIII, Marcuse, MacIntyre», in J. Horton and S. Mendus, Aspects of Toleration, cit., pp. 53-74; S. Sedley; «The Spider and the Fly: A Question of Principle», in L. Gostin, Civil Liberties in Conflict, London and NewYork, Routledge, 1988, pp. 136-144; M. Minow: ..Speaking and Writing Against Hate», Cardozo Law Review, 1990, vol. 11, pp. 1393-1408. 
se ha reivindicado legítimamente la protección del discurso racista ${ }^{43}$, no implica en absoluto, tampoco en la perspectiva diacrónica aquí asumida, el reconocimiento de la tendencia a la superación del principio antirracista (y mucho menos el deseo de que se supere). Pero implica sin duda la superación de la concepción del antirracismo como un prejuicio, $\mathrm{y}$, al mismo tiempo, comporta la superación de otro prejuicio: el que va contra el discurso ${ }^{44}$, contra la perspectiva dialógica.

Tolerar el discurso racista (admitiendo que se pueda concebir) ${ }^{45}$ no implica en modo alguno dejar de considerar el racismo intolerable.

\section{Lo intolerable}

5.1.

Llegamos así al tercer y último punto, que es el del análisis del tercer elemento, consistente en la asunción de la legitimidad del juicio de intolerabilidad.

La problemática de lo intolerable es la clásica problemática de los límites de la tolerancia. Ya he anticipado que, a los fines de una idónea caracterización de la concepción de la tolerancia que propongo, me interesa particularmente el tema de la distinción entre límites de principio y límites de hecho. En este sentido, voy a mantener la tesis de la necesidad teórica de límites de principio a la tolerancia y de la ilegitimidad de principio de límites de hecho.

${ }^{43}$ En 1977, en Skokie, suburbio de Chicago habitado por unos 40.000 judíos, muchos de ellos sobrevivientes de los campos de concentración, un grupo de extrema derecha autocalificado como «nazi», manteniendo que tenía derecho para ello, intentó organizar una manifestación portando la esvástica y con uniformes que recordaban los del ejército alemán en la segunda guerra mundial. ¿Se debía consentir la manifestación? La literatura sobre el tema es bastante amplia: Cfr. A. Neier: Defending My Enemy, New York, Dutton, 1979; D. Hamlin: The Nazi/Skokie Conflict: A Civil Liberties Conflict, Boston, Beacon Press, 1980; D. Down, Nazis in Skokie, London, University of Notre Dame Press, 1985; L.C. Bollinger: The Tolerant Society, cit.; N. Dorsen: «Is There A Right to Stop Offensive Speech? The Case ot the Nazis at Skokie», in L. Gostin, Civil Liberties in Conflict, cit., pp. $122-135$.

${ }^{44} \mathrm{El}$ término se usa aquí en el sentido fuerte de la «Diskursethik», es decir, de la ética del discurso, o argumentación racional.

${ }^{45}$ Digo «admitiendo que se pueda concebir» porque «der Diskursethik zufolge darf eine Norm nur dann Geltung beanspruchen, wenn alle von ihr móglicherweise Betroitenen als Teilnehmer eines praktischen Diskurses Einverstandnis darüber erzieln (bzw. erzielen würden), daB diese Norm gilt» (J. Habermas: MoralbewuBtsein und kommunikatives Handeln, Frankfurt a. M., Suhrkamp, 1983, p. 76). 
La primera parte de la tesis es banal y aceptada casi unánimemente, al igual que está reconocido casi unánimemente que la intolerancia es intolerable, que la intolerancia es el límite fundamental de principio, necesario para que la tolerancia no se autodestruya. Por lo tanto, no le dedicaré mucho espacio. Sin embargo, ya que se expresa con bastante frecuencia el pesar por el hecho de que del rigor teórico de la tesis no se deduzca también una fácil aplicación en la práctica, propongo algunas precisiones que en mi opinión consiguen iluminar un poco el origen de las dificultades (dificultades que en esta sede me limitaré a exponer sin intentar siquiera resolverlas). Para ello utilizaré de nuevo la preciosa distinción de Garzón Valdés entre tolerancia horizontal y vertical, enriqueciéndola esta vez con la otra distinción entre tolerancia (tanto horizontal, como vertical) pública y privada. Ni la tolerancia pública ni la tolerancia privada son explícitamente definidas por Garzón, pero de su texto es fácil inferir que la tolerancia vertical es pública cuando tiene que ver con relaciones de poder político, y la tolerancia horizontal es pública cuando concierne a la constitución del poder político mismo ${ }^{46}$.

En relación a la determinación de lo intolerable me parece de importancia fundamental subrayar:

1. Que, en el sentido de la tolerancia vertical pública, lo que es intolerable, y que, por tanto el poder no puede y no debe tolerar so pena de destruir el sentido mismo de la tolerancia, es la intolerancia vertical, tanto pública como privada, de cualquier forma que se manifieste, bien sea mediante actos, o bien mediante meras expresiones de opinión.

2. Que, en cambio, lo que un poder tolerante (sea público o privado) puede y debe tolerar son las meras expresiones de opinión, por muy intolerantes que éstas sean, dirigidas por individuos o grupos a otros individuos o grupos iguales o superiores a ellos (es decir, con los que los primeros mantengan una relación horizontal, o vertical pero de tipo ascendente).

${ }^{46}$ «La Patente de Tolerancia dictada por el emperador José II el 20 de octubre de 1781 que estableció la igualdad civil de católicos y no católicos, a la vez que abolió la censura eclesiástica -es un ejemplo de un acto de tolerancia vertical pública. También la Carta sobre la tolerancia de John Locke, escrita en 1685, se centra en casos de tolerancia vertical pública. Un buen ejemplo de tolerancia horizontal pública es el contrato social hipotéticamente supuesto como remedio a los costos de la anarquía. Si se quiere recurrir a un ejemplo de tolerancia horizontal pública no meramente supuesto sino real, puede pensarse en la tolerancia recíproca garantizada con la fundación de la Confederación Suiza después de la batalla de Morgarten (1315) por parte de los representantes de los cantones de Uri, Schwyz y Unterwalden o con el acuerdo de los «Pilgerfathers» en el «Mayflower» (1620) con miras a crear un «cuerpo político civil». (E. Garzón Valdés, op. cit., p. 18). 
3. En cambio, el problema de cómo tratar hipótesis de intolerancia horizontal privada que se manifiesten en actos, y no en meras expresiones de opinión, parece que nada tiene que ver con la temática ético-política de la tolerancia vertical pública, sino con la específicamente jurídica de la imposición de obligaciones y atribución de facultades o derechos a los individuos particulares.

El problema de los límites de la tolerancia, pues, tal como resulta de la determinación de los criterios que hemos visto, se refiere al tema de los límites del poder. Dicho esto, hay que reconocer que los orígenes de las dificultades de aplicación de tales criterios se encuentran en el hecho de que:

1. Por lo que hace al primer punto, no puede evidentemente considerarse legítimo que el poder, por respeto al principio de los límites de la tolerancia, suspenda los derechos políticos de los ciudadanos cuando tema, aunque sea del todo razonable, que del ejercicio de éstos puedan derivar amenazas para la propia tolerancia ${ }^{47}$. El método que la moderna doctrina jurídico-política ha elaborado con la finalidad de evitar actos de este tipo, desastrosos para la libertad política, es el de incluir en las Constituciones normas fundamentales de principio sustraídas a la disponibilidad de futuras mayorías parlamentarias.

2. Por lo que se refiere al segundo punto, no resulta siempre fácil establecer si entre los individuos o grupos se da una relación de tipo horizontal o vertical. Además, ¿qué tipos de poder hay que tomar en consideración? Se podría pensar, por ejemplo, que el poder de formar la opinión pública hay que tenerlo hoy particularmente bajo control, para que no genere actitudes y comportamientos intolerantes; pero el «tener bajo control la información» ¿no es una de las manifestaciones más típicas de intolerancia?

3. Respecto al tercer punto, cabe preguntarse: ¿es tolerable que los particulares, por respeto a su libertad contractual, lleven a cabo actos, o realicen comportamientos de omisión (por ejemplo, el rechazo a estipular contratos de locación), que sean expresiones de intolerancia y que tengan, supongamos, efectos de tipo discriminatorio? ¿Y cómo debe un poder político que profese y practique la tolerancia, administrar estas situaciones?

${ }^{47}$ Resulta obligado referirse aquí a la situación que está viviendo la República de Argelia en el momento en que escribo estas líneas (enero de 1992). 
5.2.

Pasemos ahora a la consideración de los límites de hecho a la tolerancia. Que tales límites son legítimos es una tesis ampliamente difundida. Significa, simplemente, que lo que puede ser tolerado depende, al menos en parte, de las circunstancias. Que si, por ejemplo, en algunas situaciones se puede (o se debe) admitir el disenso, la crítica, la protesta, no ocurre así en todas las situaciones; que si en ciertas emergencias o coyunturas se puede (o se debe) dejar camino libre a los comportamientos y a las manifestaciones de actitudes o estilos de vida distintos de los propios o de los aprobados por los grupos numéricamente mayoritarios o que detentan el mayor poder, no ocurre así en toda emergencia o coyuntura; que si en determinados contextos se puede (o se debe) tolerar la expresión de cualquier creencia, opinión o fe, no ocurre así en cualquier contexto ${ }^{48}$.

En relación a la tesis de la legitimidad de límites de hecho a la tolerancia, la primera cuestión a destacar es que presupone una caracterización de la tolerancia no implicada en ninguna de las configuraciones de la misma examinadas hasta ahora: la tolerancia no es un valor del que deriva un compromiso ético-político, sino una concesión que tal como se da, puede revocarse ${ }^{49}$. Y esto es suficiente, por sí mismo, para colocar esta especie de «tolerancia» fuera de la concepción que he propuesto.

La eventualidad de que las condiciones de revocación sean generalmente estables, y sobre todo ex ante no sólo ex post, no es suficiente para reducir significativamente la gravedad de la pérdida derivada de tal caracterización, puesto que el hecho condicionante no puede concebirse como un mero evento natural, sino que es la calificación (genéricamente social o específicamente jurídica) de una situación o de un evento, calificación realizada por el propio sujeto que después derivará de ella la suspensión de la tolerancia.

Se considera casi un tópico que el contexto en el que la tolerancia puede suspenderse legítimamente es el estado de guerra ${ }^{50}$. Cuando sucede que la diversidad entre nosotros y los demás es (de forma real

${ }^{48}$ Cfr. L.C. Bollinger, op. cit, p. 189: «Free speech is and must be an institution with a sensitivity to context $[\ldots] »$

${ }^{49}$ «La palabra tolerancia me parece en cierto modo tiránica, ya que la autoridad que tolera podría también no tolerar» (H.-G.V. Riqueti, conde de Mirabeau: Sur la liberté des cultes, Assembleé Nationale, séance du 22 août 1789). En realidad esta caracterización puede colocarse, si bien de forma contingente y no necesaria, junto a la concepción de la tolerancia como abstención de la represión, justificada por la oportunidad y no por los principios: cfr. supra, nota 9.

${ }^{50}$ Cfr. supra, nota 14. 
o percibida) tan radical que nos convierte en enemigos, internamente no podemos contentarnos con menos que con una cohesión total ${ }^{51}$.

Expresada en estos términos, esta tesis se revela ya autocontradictoria. La tolerancia y la guerra son evidentemente incompatibles. Estar en guerra significa tener un enemigo; y el enemigo, por definición, es el otro, el distinto, el extraño que representa por eso mismo un disvalor, al que se niega y se rechaza sólo por ser tal; en suma, el enemigo se define exactamente como aquel que no puede ser tolerado ${ }^{52}$. El enemigo no puede ser tolerado por definición, no ya en virtud de una razón.

Ser intolerantes significa precisamente esto: aceptar -o por convicción o por convención (o sea, por pereza)- tener, o poder tener, enemigos. Tener no ya adversarios, con los que se sea hostil por imprescindibles razones de principio, razones sobre las cuales se está dispuesto a confrontarse libremente, razones que siguen siendo imprescindibles sólo porque, y hasta que, pasan la criba de la libre discusión racional; sino tener enemigos, que quiere decir individuos que hay que tratar sólo como objetos, individuos a los que hay que obstaculizar, excluir, combatir, humillar y, si es posible, suprimir.

${ }^{51}$ Cfr. L-C. Bollinger, op. cit, p. 189: «At such times [i. e. when a nation is engaged in a full-scale war] the nation becomes like a family, and it is a matter of personal honor to commit oneself to some grater degree than usual uncriticaly to the community's cause. I exaggerate, but only to convey a ful sense of the thinking likely to he present at such times. One need only to look at the conflicts created by dissent in societies recently engaged in war -Britain in the Falklands and Israel in Lebanonto see the divisiveness and the anger stimulated by continual dissent (though I use these not as exemples of appropriate occasions for putting limits on speech but as illustrations of the kind of tensions and internal conflicts that must be taken into account in deciding when to tolerate limits)». He citado por entero el párrafo para que quede claro el embarazo que tiene Bollinger, inmediatamente después de su caracterización de la nación en guerra como familia cuyos miembros suspenden voluntariamente el juicio crítico para unirse en defensa de la causa común. ¿Puede hallarse un ejemplo más evidente de que la aceptación de la guerra lleva, inevitablemente, a la defensa de la intolerancia?

52 «El significado de la distinción de amigo y enemigo es el de indicar el extremo grado de intensidad de una unión o de una separación, de una asociación o de una disociación; ella puede subsistir teórica y prácticamente sin que, al mismo tiempo, deban ser empleadas todas las demás distinciones morales, estéticas, económicas o de

otro tipo. No hay necesidad de que el enemigo político sea moralmente malo, o estéticamente feo; no debe necesariamente presentarse como competidor económico y tal vez puede parecer ventajoso concluir negocios con él. El enemigo es simplemente el otro, el extranjero y hasta a su esencia que sea existencialmente, en un sentido particularmente intensivo, algo otro o extranjero, de modo que, en el caso extremo sean posibles con él conflictos que no puedan ser decididos ni a través de un sistema de normas preestablecidas ni mediante la intervención de un tercero 'descomprometido' y por eso 'imparcial'». (C. Schmitt: El concepto de lo 'político', Buenos Aires, Folios Ediciones, 1984, p. 23). 
Asumir una actitud tolerante es una vía, un método, un modo de relacionarse con los demás alternativo a combatirlos. Históricamente, la tolerancia se ha invocado, ora por razones ideales de principio, ora por razones contingentes de oportunidad, como alternativa a la lucha, en particular como alternativa a los horrores de la guerra ${ }^{53}$. Por tanto, suena trágicamente paradójico que el estado de guerra pueda ser considerado una circunstancia en la cual quepa suspender legítimamente la tolerancia.

En la concepción aquí mantenida, la tolerancia se justifica por el principio moral del igual respeto a las personas, que bien puede ser considerado una versión del principio kantiano en base al cual se deben tratar siempre a los hombres como fines y no como medios. La tesis de la limitación de la tolerancia en caso de guerra es, lógicamente, un absurdo parejo al de la impugnación del principio kantiano basada en la constatación del hecho de que las guerras existen y no provocan escándalo. Una impugnación similar fue la mantenida por Oliver W. Holmes, quien en 1915 expresó este tipo de reacción frente al principio kantiano: «Confieso rebelarme al instante. Cuando enrolamos a reclutas, los enviamos al frente con las bayonetas a la espalda a morir por una causa en la que tal vez no creen. Al enemigo no lo tratamos ni siquiera como un medio, sino como un obstáculo a eliminar. Ni en uno ni otro caso tengo remordimientos de conciencia y naturalmente no me resulta fácil aceptar una teoría a la que una práctica que apruebo parece contradecir» ${ }^{54}$.

El problema planteado correctamente no nos permite, pues, concebir la guerra como una situación en la que sea legítimo limitar la tolerancia, puesto que la práctica de la tolerancia no puede dejar de concebirse, y así se ha hecho a lo largo de la historia del pensamiento filosófico-político occidental, o como una elección de oportunidad con la finalidad de evitar la guerra, o como un principio por respeto al cual se debe limitar (y tendencialmente eliminar) la guerra. Así, pues,

${ }^{53}$ N. Bobbio: «Le ragioni della tolleranza», cit., p. 240, habla de la «elección de un verdadero método universal, o que debería valer universalmente, de convivencia civil: la tolerancia puede representar la elección del método de la persuasión respecto al de la fuerza o de la coacción. [...] la tolerancia como implicadora del método de la persuasión ha sido uno de los grandes temas de los mejores ensayos que han contribuido al triunfo en Europa del principio de tolerancia al fin de las sanguinarias guerras de religión». Sin embargo, Bobbio considera esta elección como una tercera razón de la tolerancia, junto a la prudencial y a la de principio. A mí, en cambio, me parece que se trata de la elección de un método, que puede llevarse a cabo o por razones prudenciales o por razones de principio.

${ }^{54}$ O. W. Holmes: «Ideals and Doubts», Illinois Law Review, 1915, pp. 1-4; tr. it. en O. W. Holmes, Opinioni dissenzienti, cit., p. 336. 
a la elección en favor de la tolerancia, sea elección de un principio o de un mero instrumento, va indisolublemente unida la tesis de la inaceptabilidad de la guerra: la guerra es -simplemente por definición- intolerable.

En consecuencia, resulta que sobre la base de la concepción aquí propuesta, actualmente tomar posición y actuar en favor de la tolerancia significa también tomar posición y actuar contra la guerra, contra la concepción de la admisibilidad, de la legitimidad de la guerra. No hay que olvidar, como ya dije, que los costes de la tolerancia son altos, muy altos. Y también pueden serlo los costes de la reivindicación de la tolerancia: mayores cuanto mayor es la fuerza del acto lingüístico de reivindicación, es decir, cuanto más necesaria sea la acción de reivindicación.

La reivindicación particular de tolerancia que propongo para la actualidad -dada la concepción de la tolerancia aquí mantenida- la propongo como la acción en que la cultura jurídica debe comprometerse si quiere ser fiel a los principios y a los valores que proclama. Esto quiere decir que tal acción no comporta costes particularmente altos en el plano jurídico-formal, porque en este plano la batalla ya se ha ganado casi en todo el mundo. Las Constituciones, los textos jurídicos supremos que expresan los ideales jurídicos de nuestro tiempo y de nuestra cultura, contienen el compromiso en favor de la tolerancia explícitamente declarada instrumental en relación a la finalidad primaria de evitar la guerra $^{55}$.

En cambio, en el plano cultural, en el de la mentalidad, en el de las opiniones corrientes, y también, lo cual es más grave, en el de la opinión común de los juristas y de los operadores jurídicos, la tesis de la inadmisibilidad (del propio carácter inconcebible de la legitimidad) de la guerra es todavía, desgraciadamente, una posición de vanguardia. La consecuencia es doble: por un lado, al ser el derecho no un hecho, sino el fruto de una operación de reconocimiento llevada a cabo fundamentalmente por los operadores jurídicos $^{56}$, la asunción formal del fin de la tolerancia y del repudio formal de la guerra, corren el riesgo de permanecer como mero flatus vocis. Por otro lado, la denuncia de la violación de la prohibición jurídico-formal de la guerra corre el riesgo

${ }^{55}$ En el Preámbulo de la Carta de las Naciones Unidas puede leerse: «WE THE PEOPLES OF THE UNITED NATIONS DETERMINED to save succeding generations from the scourge of war, which twice in our lifetime has brought untold sorrow to mankind [...] AND FOR THIS ENDS to practise tolerance and live together in peace with one another...».

${ }^{56}$ Véase la problemática relativa al «aspecto interno de las reglas» de H.L.A. Hart, The Concept of Law, Oxford, Clarendon Press, 1961. 
de no alcanzar siquiera a expresarse como crítica abierta, ya que la considerada legitimación de la intolerancia por parte de la guerra, según una opinión extendida aunque inexpresada o incluso inconsciente, puede comportar el efecto de deslegitimar la crítica ${ }^{57}$. fáciles.

Pero los teóricos de la tolerancia siempre han sabido transitar por senderos ni cortos ni

(Trad. de Josep M. Vilajosana)

${ }^{57}$ Precisamente a lo irrazonable de opiniones de este tipo se refiere el siguiente lúcido pasaje de un comentarista de la Constitución italiana (la cual contiene en su art. 11 el rechazo expreso a la guerra): «la guerra es un «hecho del Estado-comunidad, antes que del Estado-organización; del pueblo, antes que del aparato; coherentemente, el compromiso por la paz y el respeto a los otros pueblos [establecido en la Constitución italiana] vincula en primer lugar al Estado-comunidad, al pueblo italiano en su conjunto y a sus componentes individuales. Y el lógico corolario es que el aparato estatal no podría nunca, ni en el curso de la guerra, impedir manifestaciones populares que sean un desarrollo de aquel compromiso». (G. Ferrari: «Guerra (stato di)», in Enciclopedia del diritto, Milano, Giuffré, vol. XIX, pp. 832-833, cita en p. 831). 